$10-1-2020$

Ruptured mycotic reverse saphenous vein graft aneurysm: A late complication of mediastinitis

Yasir Bilal Khan

Shiraz Hashmi

Saulat Hasnain Fatimi

Follow this and additional works at: https://ecommons.aku.edu/pakistan_fhs_mc_surg_cardiothoracic

Part of the Cardiology Commons, Cardiovascular Diseases Commons, and the Surgery Commons 
CASE REPORT

\section{Ruptured mycotic reverse saphenous vein graft aneurysm: A late complication of mediastinitis}

Yasir Bilal Khan, Shiraz Hashmi, Saulat Hasnain Fatimi

\begin{abstract}
Reverse saphenous vein graft aneurysm (RSVGA) is a rare complication that occurs after coronary bypass grafting surgery. Mycotic aneurysm of reverse saphenous vein graft is even more rare. We describe the case of a 76-yearold man who underwent coronary artery bypass grafting 10 years back and had mediastinitis four to five months after surgery. He presented with chest pain in July 2017 and was diagnosed as NSTEMI. Angiography showed left main coronary artery disease (90\%) with aneurysm of reverse saphenous vein graft to right posterior descending artery(RPDA). Emergent percutaneous coronary intervention $(\mathrm{PCl})$ of the left main artery was performed as the patient suffered ventricular arrhythmia for which cardiopulmonary resuscitation (CPR) was done. After CPR, haematoma appeared on the left side of the sternum subcutaneously. CT scan of the chest was conducted which showed a pseudoaneurysm measuring $35 \mathrm{~mm}$ in the mid-segment of reverse saphenous vein graft (RSVG) to the right posterior descending artery (RPDA) with a surrounding mediastinal haematoma communicating with subcutaneous haematoma, so RSVGA to RPDA was coiled. As blood culture grew candida, antifungal medication was started. When the patient's condition stabilised he was discharged.
\end{abstract}

Keywords: Mycotic pseudoaneurysm, Coiling of vein graft aneurysm, Rupture graft aneurysm.

\section{DOI: http://doi.org/10.5455/JPMA.301599}

\section{Introduction}

Aneurysms of graft can develop slowly over many years and often have no symptoms. These can be either true or false aneurysms and have the tendency to increase in size with time. It was first described in 1975 by Riahi et al, ${ }^{1}$ and is still an uncommon presentation. The aetiology and mechanism of graft aneurysm is not fully understood but different risk factors have been described. They mostly present with chest pain or shortness of breath and can rupture, leading to fatal complications. Infection

Aga Khan University Hospital, Karachi, Pakistan.

Correspondence: Yasir Bilal Khan. Email: leopk78@yahoo.com contributes to early reverse saphenous vein graft aneurysm. Till 2004, only four cases were found to have mycotic aneurysm. ${ }^{2}$ In this case report we describe a patient who had CABG performed a decade ago and presented with reverse saphenous vein graft aneurysm to the right posterior descending artery aneurysm whose post-operative recovery was complicated by mediastinitis.

\section{Case Report}

A 76-year-old man with hypertension, ischaemic heart disease, and chronic kidney disease who had undergone coronary artery bypass grafting (CABG) 10 years ago presented to the ER. The initial postoperative course of CABG was complicated by mediastinitis which was treated conservatively. The patient remained asymptomatic for five years but developed localised discharge from the sternal wound which was treated by removing the sternal wire. The patient did well for next five years. He then presented to the ER with the history of right sided chest pain, shortness of breath and fever. ECG showed ST depression in lateral leads and trop-I was mildly leaking. He was treated medically for NSTEMI, acute kidney injury and urinary tract

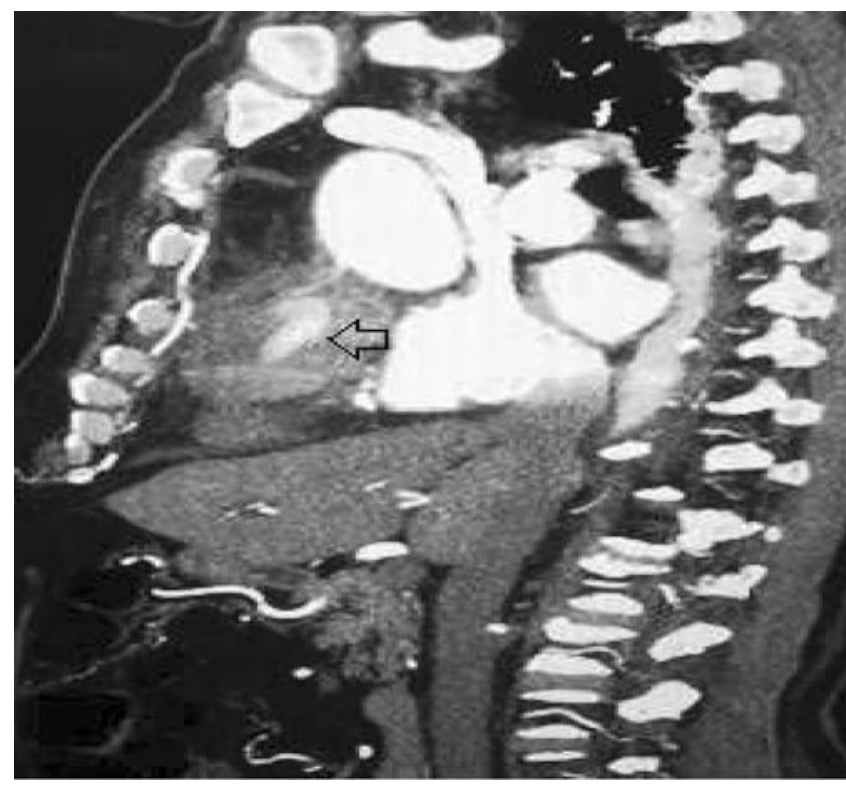

Figure-1 A: CT scan of the chest with contrast showing pseudoaneurysm of reverse saphenous graft aneurysm. 


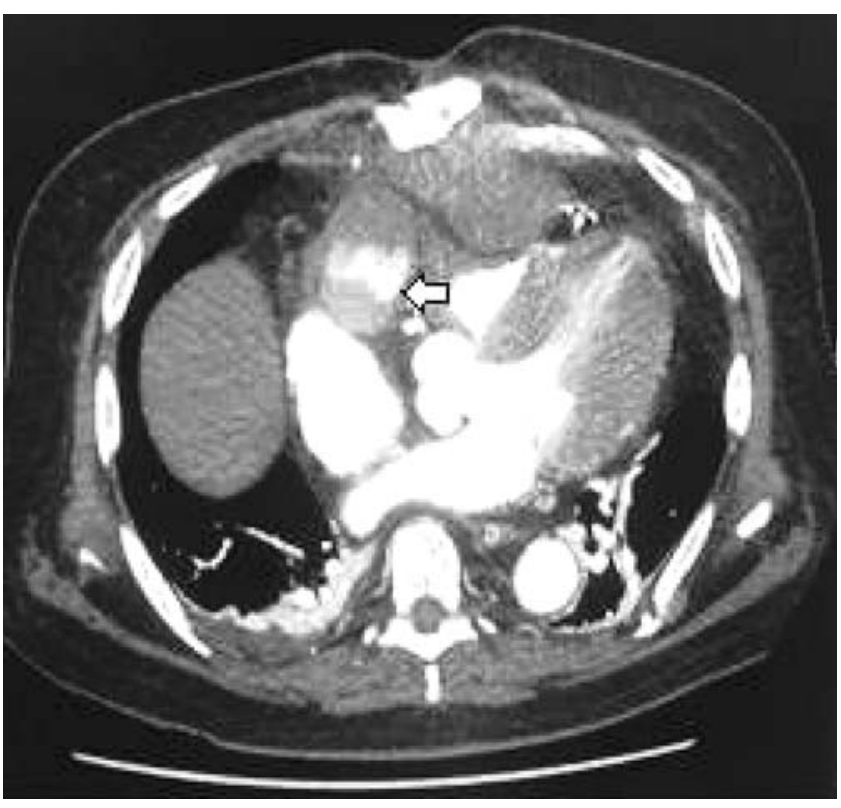

Figure-1 B: CT scan of the chest, a cross section showing pseudoaneuyrsm.

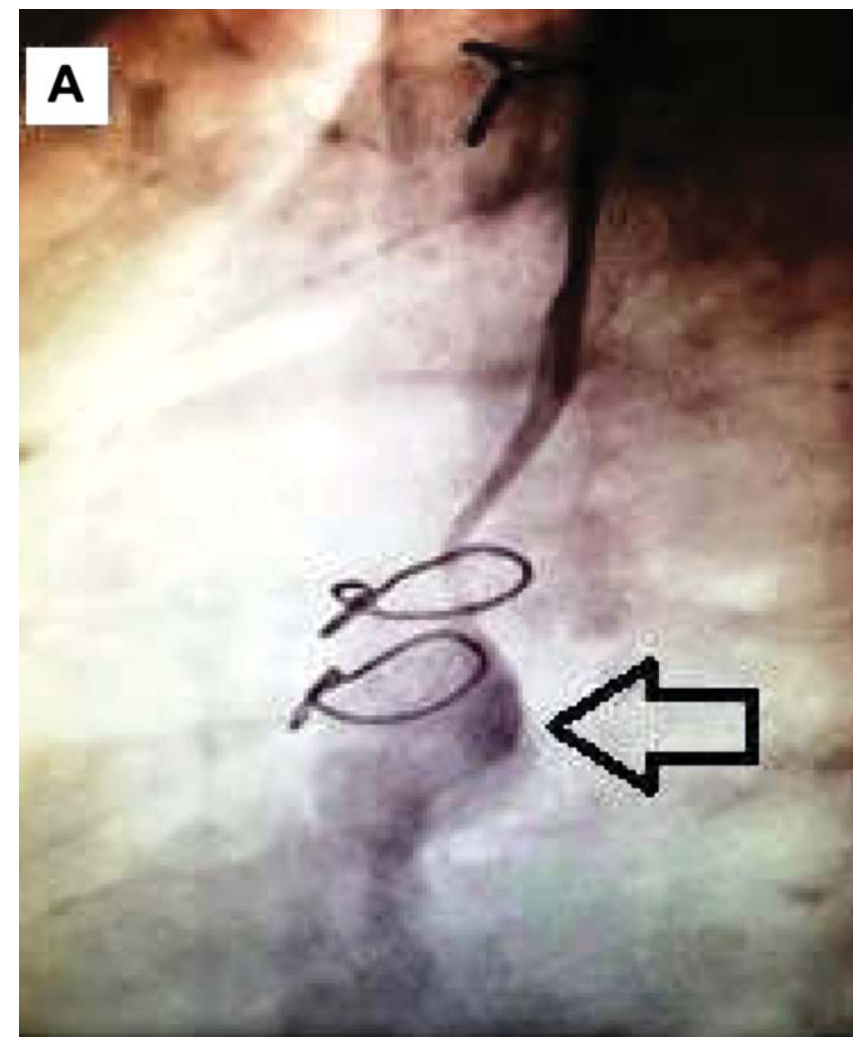

Figure-2A: Angiographic image showing aneurysmal dilation of reverse saphenous vein to posterior descending artery.

infection. The patient had a new episode of tachypnoea with chest pain during his stay at the hospital. ECG showed new changes with T-wave inversion in anterior leads, and

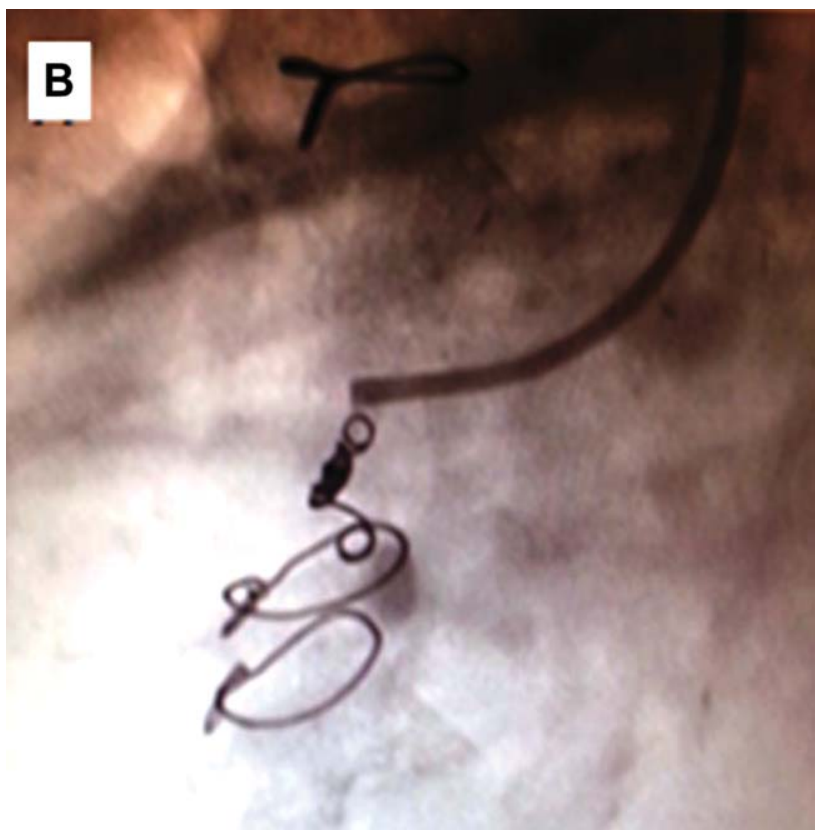

Figure-2B: Angiographic image showing coiling of reverse saphenous vein graft aneurysm to posterior descending artery.

ST depressions in lateral leads with new trop leak. A two stage angiography was planned due to his raised creatinine. The first angiography revealed $90 \%$ occlusion of the left main coronary artery, $100 \%$ proximal occlusion of the left anterior descending artery (LAD) which was distally filling from left internal mammary artery graft (LIMA) from the previous bypass surgery. The diagonal branch was filling from reverse saphenous vein graft (RSVG), the left circumflex coronary artery was normal and the right coronary artery was occluded $100 \%$ proximally. The RSVG to right posterior descending artery (RPDA) was $100 \%$ occluded with a mid-segment pseudoaneurysm (Figure2A). The patient continued to have chest pain and fever. He developed polymorphic Ventricular Tachycardia and was given two minutes of cardio pulmonary resuscitation (CPR). After CPR, a mass was noted on the left side of the sternum with serosanguineous discharge. CT scan showed $35 \mathrm{~mm}$ pseudoaneurysm of mid-segment RSVG to RPDA graft and a haematoma was identified on the front of the heart measuring $107 \times 50 \mathrm{~mm}$, extending to subcutaneous tissue just anterior to the manubrium which measured $46 \times 25$ mm (Figure-1A and 1 B). On Ultrasound Doppler Scan of the chest, no colour flow was seen. Blood culture grew candida, while the rest of the culture was negative. Percutaneous coronary intervention $(\mathrm{PCl})$ to the left main artery was performed due to continuing chest pain and ventricular arrhythmias. After $\mathrm{PCl}$ the patient's symptoms improved. As CT scan showed a haematoma near the aneurysm which was an indication of a ruptured aneurysm, 
so it was decided to coil the aneurysm (Figure-2B). The procedure was uneventful, and the patient remained stable. The patient was managed as mycotic pseudoaneurysm and was discharged on oral anti-fungals.

\section{Discussion}

Reverse saphenous vein graft aneurysm (RSVGA) is a rare post coronary artery bypass grafting (CABG) complication with a frequency of $0.07 \% .^{2}$ Patients with RSVGA mostly present with chest pain, shortness of breath, MI, heart failure, arrhythmias, shock and haemoptysis or as incidental finding on imaging. RSVG mostly occurs 10 years after CABG. Males are more affected $(87 \%)^{3}$ than females. Early RSVGA occurring within one year after CABG are attributed to technical problems, ${ }^{4}$ infection, $, 3,5$ intrinsic weakness of the vessel wall, 6 while late presentation is mostly attributed to atherosclerotic changes in the vessel wall.7 Like aneurysm of other vessels, RSVGA has the tendency to grow and the rate of growth varies widely. ${ }^{8}$ Management of RSVGA is conservative, surgical and percutaneous depending on the site, size and location of the aneurysm. Dieter et al, ${ }^{2}$ retrospectively reviewed 13 patients with SVGAs, of which two were treated surgically and 11 were managed conservatively, but it does not show any significant difference between both interventions. Considering the risk of rupture, embolization, myocardial infarction and death, Kalimi $\mathrm{R}$ et al, ${ }^{9}$ believe that these aneurysms should be surgically resected and the coronary artery distal to the aneurysm should be revascularized. Sareyyupoglu B et al ${ }^{10}$ studied 16 SVGA repair patients and proposed that surgical revascularisation should be recommended for symptomatic patients with SVGAs of $>1$ $\mathrm{cm}$ diameter or if graft flow is diminished. Memon A-Q11 recommended surgical resection and revascularisation in addition to coil embolization as a viable option in cases of high surgical risk. A literature review by Ramirez FD et al,3 found surgical management of SVGAs in $58.4 \%$ cases with either aneurysmal resection or ligation followed by bypass grafting in cases with significant myocardium to be in danger. During the last decade percutaneous intervention (with coil embolization, Amplatzer vascular occlusion, or covered stent placement) was reported in only $15.8 \%$ cases and conservative management was used in $20.1 \%$ cases.

In our case, we found mediastinitis to be a profound factor, while RSVGA may appear as a late complication of mediastinitis. Our case signifies two factors, one that mediastinitis can lead to late RSVGA aneurysm and second, if the culprit vessel is not the aneurysmal graft, it is better to coil and block the aneurysm because it can rupture, as was observed in this case, which can eventually be a lethal complication. Case specific preoperative planning and selection of the appropriate technique can improve the outcome. Careful follow up with CTA or MRI is warranted after such repairs to reduce the related morbidity and mortality.

\section{Conclusion}

From this case report it is concluded, that an aneurysm of the vein graft after coronary artery bypass surgery, should be intervened through coiling so as to reduce the risk of rupture.

Consent: Patient's consent was taken for publishing the case report.

Disclaimer: None to declare.

Conflict of Interest: None to declare.

Funding Sources: None to declare.

\section{References}

1. Riahi M, Vasu CM, Tomatis L, Schlosser R, Zimmerman G. Aneurysm of saphenous vein bypass graft to coronary artery. J Thorac Cardiovasc Surg. 1975; 70:358-9.

2. Dieter RS, Patel AK, Yandow D, Pacanowski JP, Bhattacharya A, Gimelli G, et al. Conservative vs. invasive treatment of aortocoronary saphenous vein graft aneurysms: treatment algorithm based upon a large series. Vascular. 2003; 11:507-13.

3. Ramirez FD, Hibbert B, Simard T, Pourdjabbar A, Wilson KR, Hibbert R, et al. Natural History and Management of Aortocoronary Saphenous Vein Graft Aneurysms A Systematic Review of Published Cases. Circulation. 2012; 126:2248-56.

4. Kallis P, Keogh BE, Davies MJ. Pseudoaneurysm of aortocoronary vein graft secondary to late venous rupture: case report and literature review. Br Heart J. 1993; 70:189-92.

5. Douglas B, Bulkley B, Hutchins G. Infected saphenous vein coronary artery bypass graft with mycotic aneurysm. Fatal dehiscence of the proximal anastomosis. Chest. 1979; 75:76-7.

6. Ennis BM, Zientek DM, Ruggie NT, Billhardt RA, Klein LW. Characterisation of a saphenous vein graft aneurysm by intravascular ultrasound and computerized three-dimensional reconstruction. Cathet Cardiovasc Diagn. 1993; 28:328-31.

7. Vlodaver Z, Edwards JE. Pathologic changes in aortic-coronary arterial saphenous vein grafts. Circulation. 1971; 44:719-28.

8. Hickey EJ, Velissaris T, Tsang GM. A saphenous vein graft aneurysm with fistula development to the right atrium: surgical management of a rare bypass graft complication. Can J Cardiol. 2008; 24:915-6.

9. Kalimi R, Palazzo RS, Graver LM. Giant aneurysm of saphenous vein graft to coronary artery compressing the right atrium. Ann Thorac Surg. 1999; 68:1433-7.

10. Sareyyupoglu B, Schaff HV, Ucar I, Sundt TM, Dearani JA, Park SJ. Surgical treatment of saphenous vein graft aneurysms after coronary artery revascularization. Ann Thorac Surg. 2009; 88:1801-5.

11. Memon A-Q, Huang RI, Marcus F, Xavier L, Alpert J. Saphenous vein graft aneurysm: case report and review. Cardiol Rev. 2003; 11:26-34. 